\title{
Morphological, immunohistochemical and miRNA21 expression manifestations related to cellular apoptosis in focal cerebral ischemia in a rat alcoholism model
}

\author{
D.P.C. Tirapelli ${ }^{1}$, M.L.A. Cirino ${ }^{1}$, J.P. da Silva ${ }^{1,2}$, I.S. Gula ${ }^{1,3}$, \\ M.F.G.S. Tazima ${ }^{1}$, V.P.S. Fazan ${ }^{1}$, C.A.M. de Carvalho ${ }^{1,4}$, P.C. Novais ${ }^{1,5}$, \\ F.S. Lizarte Neto ${ }^{1}$, M.T. Durand ${ }^{3}$, B.C. Schimming ${ }^{6}$, C.G. Carlotti Junior ${ }^{1}$, \\ B.O. Colli ${ }^{1}$ and L.F. Tirapelli ${ }^{1}$ \\ ${ }^{1}$ Departamento de Cirurgia e Anatomia, Universidade de São Paulo, \\ Ribeirão Preto, SP, Brasil \\ ${ }^{2}$ Faculdade de Taquaritinga, Taquaritinga, SP, Brasil \\ ${ }^{3}$ Faculdade de Medicina, Universidade de Ribeirão Preto, Medicina, \\ Ribeirão Preto, SP, Brasil \\ ${ }^{4}$ Departamento de Medicina, Centro Universitário Barão de Mauá, Ribeirão \\ Preto, SP, Brasil \\ ${ }^{5}$ Programa de Pós-graduação em Reabilitação das Interações Estruturais, \\ Universidade de Marília, Marília, SP, Brasil \\ ${ }^{6}$ Departamento de Anatomia, Universidade Estadual de São Paulo, \\ Botucatu, SP, Brasil
}

Corresponding author: L.F. Tirapelli

E-mail: lab.biomol.cirurgia@fmrp.usp.br

Genet. Mol. Res. 19 (4): gmr18624

Received October 24, 2020

Accepted November 12, 2020

Published December 31, 2020

DOI http://dx.doi.org/10.4238/gmr18624

\begin{abstract}
Cerebral ischemia is one of the main causes of morbidity and mortality as Abusive use of alcohol also causes health problems, along with considerable social and economic repercussions. We examined how these two conditions affect histopathological signs, morphometrics, expression of apoptosisrelated proteins CASPASE 3 and BCL2, and serum gene expression of miRNA-21, in cerebral ischemia associated with a chronic alcoholism model in brain tissue (striatum, lateral and dorsolteral cortex). Fifty adult Wistar rats were divided into five groups: control (C), sham (S), ischemic (I), alcoholic (A) and ischemic/alcoholic
\end{abstract}


groups $(\mathrm{I}+\mathrm{A})$. Brain samples were processed for histopathological, morphological, and immunohistochemical analyses for the expression of CASPASE 3 and BCL2, and blood was collected for analysis of miRNA-21 gene expression by real-time PCR. Histopathological changes such as neurons with pyknotic nuclei and diffuse neurons with loss of the contour of their cellular membranes and cytoplasmic edema, were observed in groups I and I+A in all three areas. Protein expression of CASPASE 3 was significantly higher than that of $\mathrm{BCL2}$, with higher expression in groups $\mathrm{I}$ and $\mathrm{I}+\mathrm{A}$ for these two proteins. Serum expression of miRNA-21 was low in all groups, and was slightly higher in groups $\mathrm{I}$ and $\mathrm{I}+\mathrm{A}$, with no significant differences. The histopathological and morphometric alterations, which were observed mainly in the ischemic animals, correlated with the expression of CASPASE 3. The expression of BCL2 was greater where histopathological changes and expression of CASPASE 3 were less evident. The I and I+A groups presented more histopathological changes, with greater neuronal loss and cerebral edema, and greater expression of CASPASE 3, suggesting that ischemia and alcoholism, when associated, can cause considerable injury and damage to brain tissue.

Key words: Cerebral ischemia; Apoptosis; Alcoholism; Morphology; miRNA-21

\section{INTRODUCTION}

Cerebral ischemia is one of the main causes of morbidity and mortality, despite considerable information related to its prevention. Stroke occurrences will have an estimated cost of 240 billion dollars by 2030, according to the American Heart Association. The development of therapies that can effectively act in the prevention, containment and treatment of this disease should be a priority (Lee et al., 2018). Its cause is the interruption of cerebral blood flow, leading to oxygen and glucose deprivation (Chaitanya et al., 2010).

There are several examples of clinical situations in which the temporary focal ischemic stroke followed by reperfusion occurs. Cases of transient ischemic attacks, clamping, or temporary stenosis during neurovascular surgical procedures (David et al., 1996) can be cited. It is known that neurons can die two or three days after transient global cerebral ischemia and the characteristic infarction resulting from this ischemia has been confirmed in several studies (Kamestu et al., 2003). When persistent and critical, this impairment to blood flow may eventually lead to a cascade of biochemical events and pathological reactions leading to neuronal death. Some of them are immediate, while the reperfusion process triggers others (Hachimi-Idrissi et al., 2004). However, the mechanism responsible for late infarction is not clearly described. Although some researchers have suggested that apoptosis is the major pathophysiological mechanism, controversy remains whether apoptosis, necrosis or both would be involved in late neuronal death.

Alcohol is consumed in moderation by a large part of the population as part of social gatherings, weddings and religious ceremonies in most Western countries. However, alcohol abuse can lead to physical dependence and damage associated to target organs, 
particularly the brain and liver. Potential damages from alcohol abuse include not only the organism in its structural, cellular, and molecular form, but other levels that are beyond the individual: family, society, culture and behavior (Reilly et al., 2014).

Several studies have shown that excessive alcohol consumption mainly compromises the Central Nervous System because alcohol has an affinity with organs that have a higher concentration of water (St. Clair, 1991).Therefore, the brain is one of the organs most affected by alcohol abuse and among the consequences are biological alterations, cognitive and emotional deficiencies (Bala and Szabo, 2012).

Studies by Love (2003) and Rami (2003) have indicated that caspase activation is critical for the mechanism of apoptosis that occurs after ischemia-induced brain injury. During apoptosis, intracellular activation of the caspase cascade leads to degradation of the cellular constituents and finally, the cell death (Rami, 2003).

Recent studies have revealed that microRNAs (miRNAs) play an important role in a large number of biological processes, such as growth regulation and cell differentiation, cell proliferation, apoptosis, neuronal differentiation, neurogenesis, as well as in the presence of a variety of neuronal disorders (Kosik and Krichevsky, 2005), including alcoholism (Miranda et al., 2010) and cerebral ischemia (Dharap et al., 2009; Buller et al., 2010; Liu et al. 2010).

In this context, since little is know about how brain morphology is affected by apoptosis and miRNA-21 expression in experimental cerebral ischemia associated with alcoholism, we analyzed morphological alterations in brain tissue (striatum, lateral and dorsolateral cortex) and apoptosis through a immunohistochemical study of the expression of CASPASE 3 and BCL2 and miRNA-21 serum expression in ischemic cell death after experimental induction of transient focal cerebral ischemia with reperfusion, associated with chronic alcoholism in a rat model.

\section{MATERIAL AND METHODS}

Fifty male Wistar rats (Rattus norvegicus) weighing between 280 and 310 grams were used. The animals were divided into five experimental groups: Control Group (C): 10 animals that were euthanized without performing the surgical procedure. Sham Group (S): 10 control animals that underwent complete simulation of the surgical process, with introduction of the obstructing wire, but without occlusion of the middle cerebral artery, and then euthanized. Ischemic Group (I): 10 animals submitted to focal ischemia by occlusion of the middle cerebral artery for $90 \mathrm{~min}$, followed by reperfusion of $48 \mathrm{~h}$, and then euthanized. Alcoholic Group (A): 10 animals received only ethanol diluted to $20 \%$ in water for four weeks and then euthanized. Ischemic and alcoholic group (I + A): 10 animals that underwent the same treatment of group A and after the four week period were subjected to focal ischemia by occlusion of the middle cerebral artery for $90 \mathrm{~min}$, followed by reperfusion of $48 \mathrm{~h}$, and then euthanized.

For the animals of groups A, and A + I, the "semi-voluntary alcoholism" model was used. It was supplied through ethanol, which was the only liquid food available to these animals. For these experimental groups, a preparation was necessary where the animals were conditioned to a brief period of gradual adaptation to ethanol consumption, consisting of the supply of ethanol diluted in water with a gradual concentration, changed in increasing 
weekly doses of 5, 10 and $20 \%$ in water. The experimental phase started only after the third week of adaptation.

The animals were randomly allocated to the respective groups after a draw, and the surgical procedure for cerebral ischemia was performed by introducing a thickened end wire to interrupt the flow in the middle cerebral artery for a period of 90 min (Koiosumy et al., 1986), modified by Carlotti Jr. et al. (2001), followed by the expected reperfusion period $(48 \mathrm{~h})$. This study was approved by the Ethics Committee on Animal Experimentation of the Ribeirão Preto Medical School (protocol number 279/2005).

For the study by optical microscopy, transmission electron microscopy and immunohistochemistry, only one coronal section $(2 \mathrm{~mm})$ was selected with a section made at the height of the optic chiasm and, for ultrastructural evaluation by transmission electron microscopy, a sample was drawn from the rostral region of the coronal cut, parallel to the lateral wall of the lateral ventricle between the piriform area (inferiorly), through the striatum and ending in the dorsolateral region of the cortex (uppermost) (Figure 1).

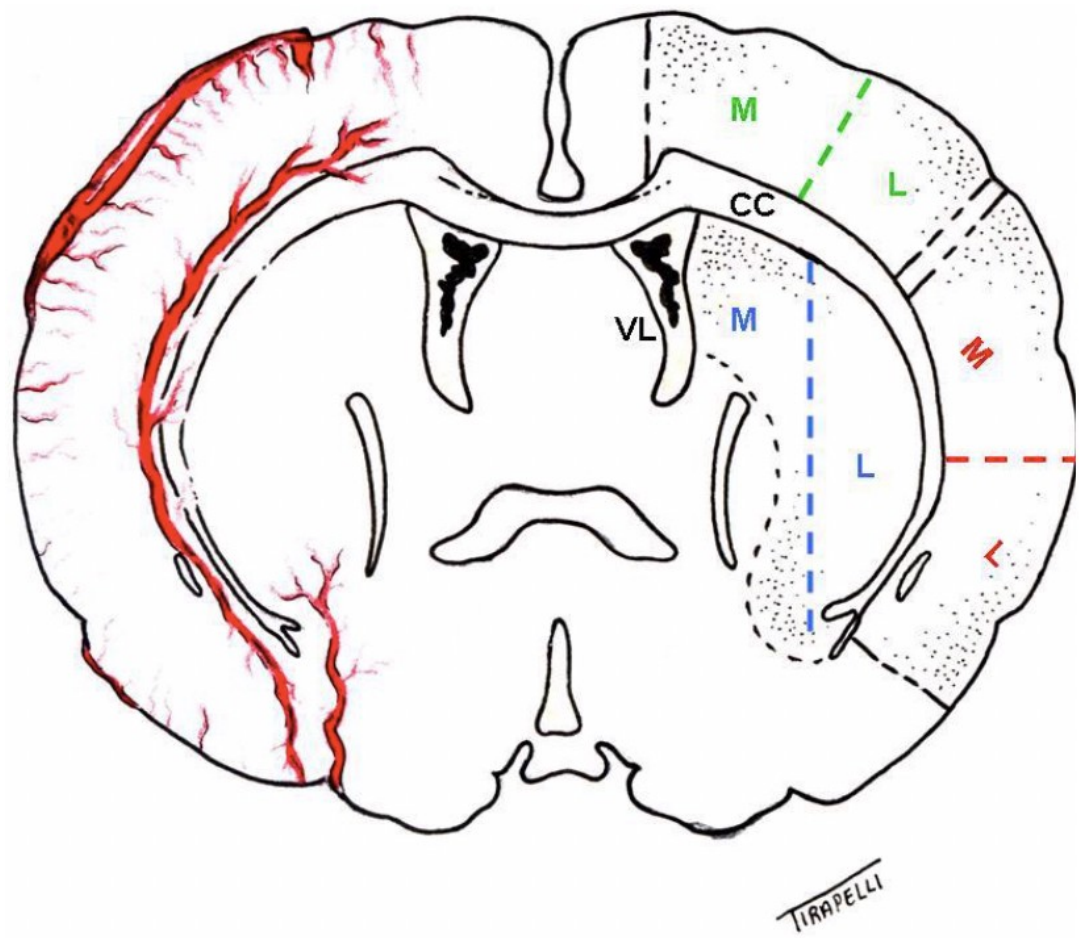

Figure 1. Coronal cortex showing the medial (M) and lateral (L) fields demarcated for the three evaluation areas in the immunohistochemical study: area 1 - dorsolateral cortex (in green); area 2 - lateral cortex (in red); and, area 3 - striatum (in blue) (CC); outer capsule; lateral ventricle (VL).

The striatum was subdivided into two fields: A) medial- below the corpus callosum and lateral to the lateral ventricle; and B) one lateral-medially to the corpus callosum and outer capsule. It is important to note that the choice of the medial field was performed not too close to the lateral ventricle, because this region of the striatum is irrigated by branches of the anterior cerebral artery. 
For histopathological analysis, the samples were stained by the Luxol Fast Blue technique. Morphometric analysis was performed by counting the number of neurons in the striatum in order to evaluate possible neuronal loss. For the immunohistochemical analysis the avidin-biotin-peroxidase method was used with analysis of the antibodies related to the mechanism of apoptosis: CASPASE 3 and BCL2. In the analysis by transmission electron microscopy, ultrastructural alterations of the cerebral tissue were observed in the cortical region and in the striatum.

Finally, analysis of the serum gene expression of miRNA-21 was performed using the real-time PCR technique through blood collected from the ventral artery of the tail. GraphPad Prism version 8.00 for Windows (GraphPad Software, San Diego - California USA) was used for statistical analysis, performed using the Kruskal-Wallis test and posttest of Dunns multiple comparison, with values of $\mathrm{P}<0.05$ being considered significant.

\section{RESULTS}

\section{Histopathological evaluation}

According to the images of Figure 2, the most evident alterations were found in the deeper layers of the cortex, in the animals of groups I and I + A, but not reaching all the extension of the dorsolateral and lateral regions and neither in the striatum. Among these findings are the presence of neurons with pyknotic nuclei, characteristic cells in the process of necrosis and diffuse neurons presenting histological aspects with characteristic lesions: loss of the contour of their cell membranes; presence of cytoplasmic edema and lighterlooking foam core. Also in these two groups, the presence of leukocyte or inflammatory infiltration characterized by the presence of neutrophils inside the capillaries, in diapedesis and with interstitial infiltration in the cortical tissue, mainly in the dorsolateral cortex, was observed.

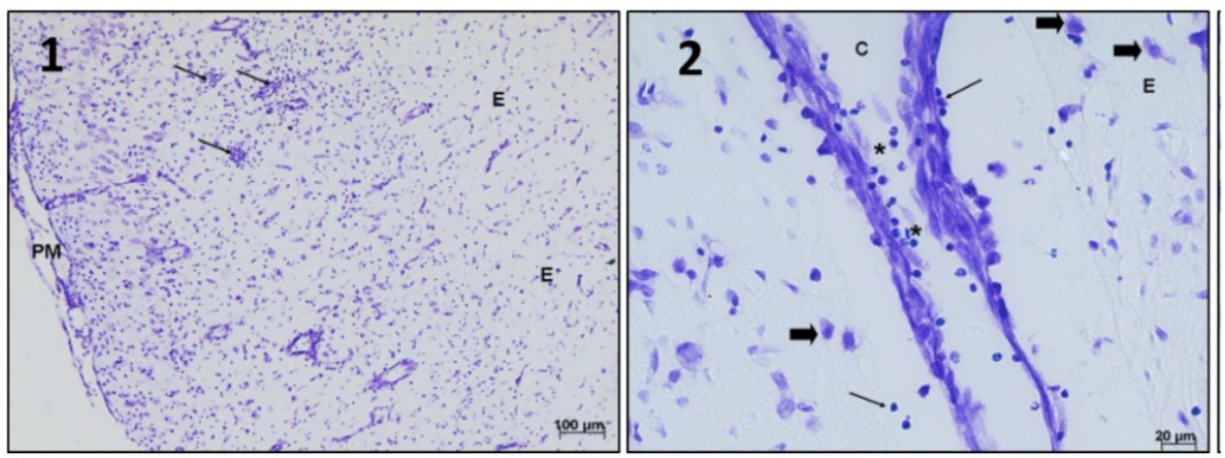

Figure 2. Photomicrographs, Luxol fast blue: 1 dorsolateral region of the frontal cortex of the I + A group: interstitial edema (E) with pyknotic nuclei and regions of inflammatory infiltration $(\rightarrow$ ) along layer III capillaries. Pia mater (PM). 100x. 2 dorsolateral region of the frontal cerebral cortex of group I: inflammatory infiltration from a central capillary, with the presence of a large number of neutrophils $\left(^{*}\right)$ in its lumen, including diapedesis and interstitial tissue $(\rightarrow)$; $(\mathrm{N})$ close to the lateral wall of the lateral ventricle $(\mathrm{E})$ and some neurons with pyknotic nuclei (photography 400x. part not irrigated by middle cerebral artery) and more laterally, region of interstitial edema (E) with the presence of pyknotic nuclei (thicker arrows) and some neutrophils $(\rightarrow) .400 \mathrm{x}$. 
For animals of group A, the same findings were found, except interstitial edema and inflammatory infiltration, which were less evident than in groups I and I + A. No histological changes were observed in the animals of group S (Figure 3).

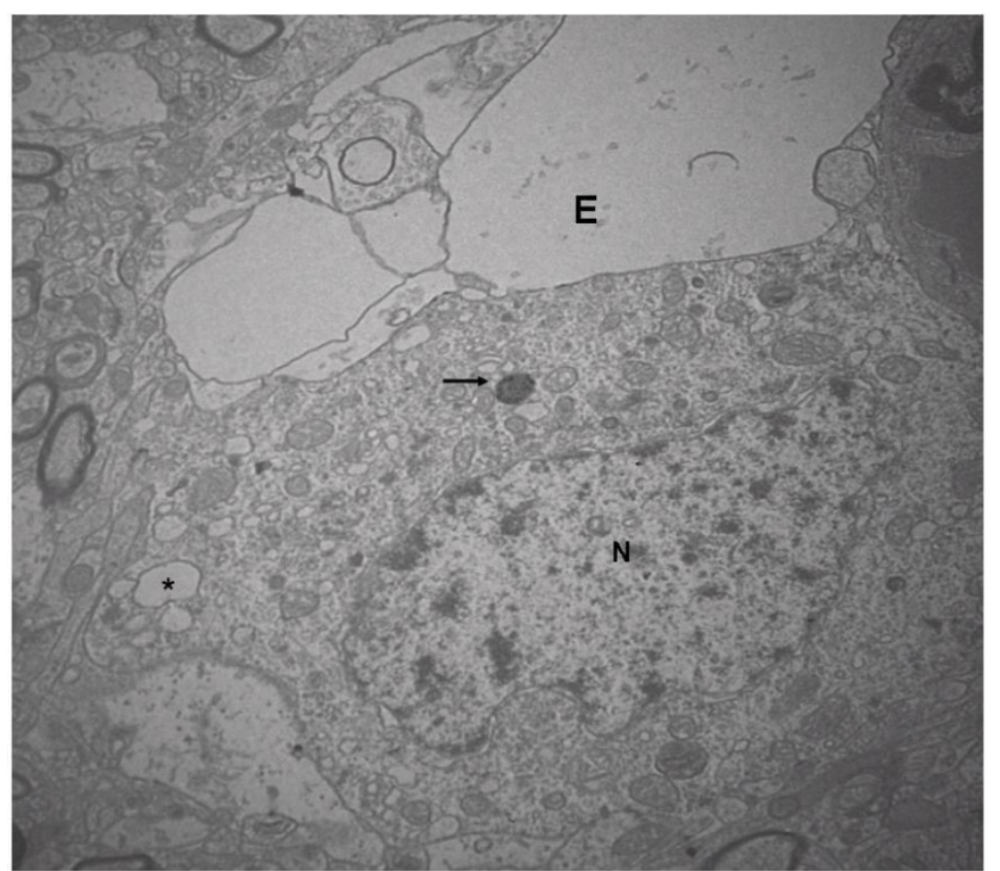

Figure 3. Electronmicrograph of cortex of an animal of group I + A with the presence of neuron with lysosome (arrow) and cytoplasmic vacuoles (*). Above, region of edema (E) .10000x.

\section{Morphometric evaluation}

In the count of the number of neurons in the striatum, Figure 4 shows the means of the total number of neurons in the striatum in each experimental group. There were significant differences $(\mathrm{P}<0.0001$, Kruskal-Wallis test $)$ between the groups: CxI $(\mathrm{P}<0.001), \mathrm{CxI}+\mathrm{A}(\mathrm{P}<0.01)$ and between SxI $+\mathrm{A}$, IxA and AxI $+\mathrm{A} \mathrm{P}<0.05)$, Dunns post-test (Figure 4).
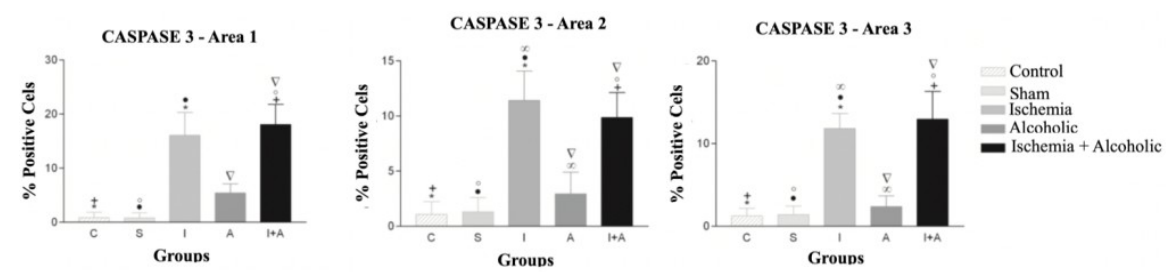

Figure 4. Mean \pm standard deviation of the protein expression of CASPASE 3: in Area 1- dorsolateral cortex between the rat groups $(\mathrm{P}<0.0001$, Kruskal-Wallis test, CxI, CxI + A, SxI, SxI + A $(\mathrm{P}<0.001)$ and AxI $+\mathrm{A}(\mathrm{P}<$ $0.05)$, Dunns post-test $).(\mathrm{P}<0.001)$ and $\mathrm{AxI}+\mathrm{A}(\mathrm{P}<0.05)$ were observed in the 2-lateral cortex area: $\mathrm{P}<$ 0.0001, Kruskal-Wallis test, CxI, CxI + A, SxI, SxI+A, Dunns post-test). $(\mathrm{P}<0.001)$, IxA, and AxI + A $(\mathrm{P}<$ 0.01 ) were observed in the 3-Striatum Area: $\mathrm{P}<0.0001$, Kruskal-Wallis test, CxI, CxI + A, SxI, SxI+A), Dunns post-test). 


\section{Immunohistochemical analysis}

Nuclear positive marking was observed, mainly in groups I and I + A, when compared to groups $\mathrm{A}, \mathrm{S}$ and $\mathrm{C}$, of both CASPASE 3 and BCL 2 proteins. CASPASE 3 is dispersed in all layers, in different areas 1, 2 and 3 and in the striatum (Figure 5). BCL2 has greater expression in area 1 (dorsolateral cortex), and in area 2 (lateral cortex) the marking can be considered moderate.
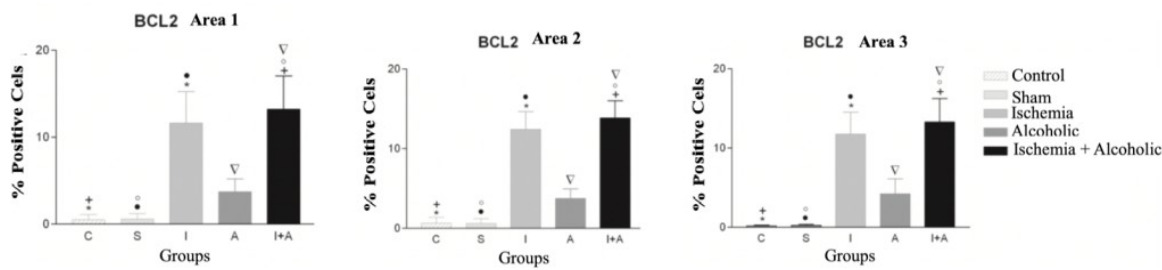

Figure 5. Mean ( \pm standard deviation) of BCL2 protein expression: Area 1- Dorsolateral cortex between the rat groups $(\mathrm{P}<0.0001$, Kruskal-Wallis test, $\mathrm{CxI}, \mathrm{CxI}+\mathrm{A}$, SxI, SxI $+\mathrm{A} \mathrm{P}<0.001)$ and AxI $+\mathrm{A}(\mathrm{P}<0.05)$, Dunns post-test). $(\mathrm{P}<0.001)$ and $\mathrm{AxI}+\mathrm{A}(\mathrm{P}<0.05)$, post-morbidity between the studied groups $(\mathrm{P}<0.0001$, KruskalWallis test, CxI, CxI + A, SxI, SxI +A- Dunns post-test). $(\mathrm{P}<0.001)$ and AxI + A $(\mathrm{P}<0.05)$, Dunns post-test $)$.

When compared to the labeling of CASPASE 3 and BCL2 in the lateral cortex, it was observed that it was slightly larger for the BCL2 protein in groups A, I and I+ A.

In the striatum, little cytoplasmic positive marking for the BCL2 protein was diffusely observed in groups I and I + A, when compared to groups A, S and C.

\section{Analysis of the gene expression of miRNA-21}

The serum expression of miRNA-21, shown in (Figure 6), shows low expression in all groups, and was slightly higher in the animals of groups I and I + A, with no significant difference $(\mathrm{P}=0.2637$, Kruskal-Wallis test $)$.

\section{mRI-21 Gene Expression}
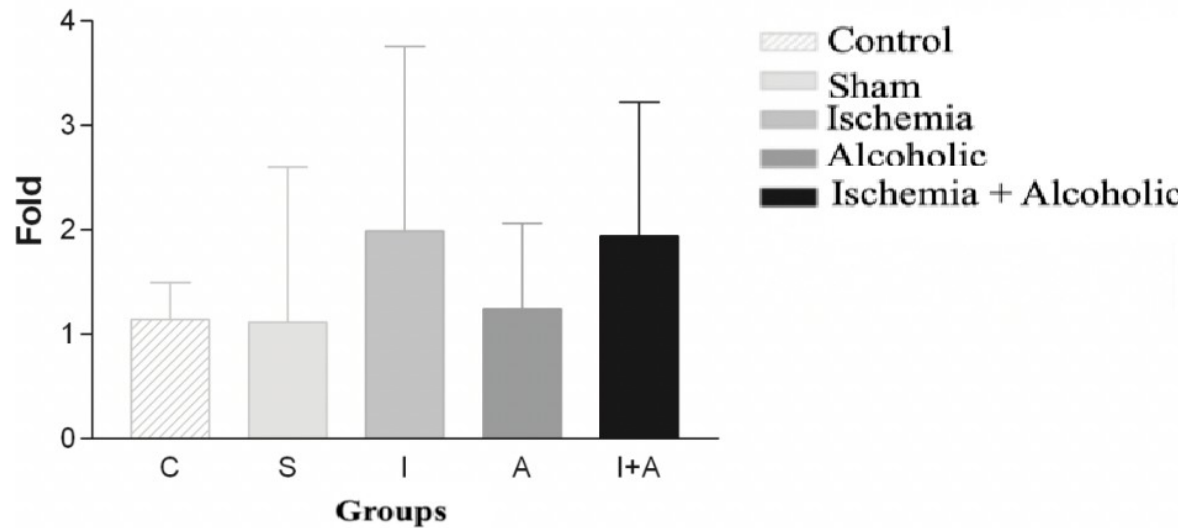

Figure 6. Mean \pm standard deviation of the miRNA-21 serum gene expression between the rat groups (CxI, CxI + A) $(\mathrm{P}=0.2637$, Kruskal-Wallis test $)$. 
No significant differences were observed between the groups in the glycemia, hemoglobin and hematocrit rates, and the blood gas values varied within the parameters established for the experiment.

\section{DISCUSSION}

There are several well-established animal models for the study of focal and global cerebral ischemia. In our study, we used the model of induction of temporary focal ischemia by occlusion of middle cerebral artery with intraluminal wire (Koizumi et al., 1986; Carlotti Jr. et al., 2001), since it is a well- established and reliable model, widely used in studies involving the subject, and that allows the possibility of reperfusion.

In all cases, ischemia causes dysfunction or loss of brain cells. In focal cerebral ischemia, most ischemic focus cells undergo necrosis with swelling and rupture of the cellular organelles. The margin of the brain tissue that is hypo-perfused around the ischemic focus is called ischemic penumbra and has a capacity for recovery if the perfusion is improved (Ginsberg, 2003). Cell death in the penumbra is considered as an active process, mainly dependent on the activation of the program of cell death or apoptosis (Ferrer, 2003).

In the morphological evaluation of the lesions caused by cerebral ischemia, several techniques have been used. The study of cortical neurons and striatum after focal ischemia in rats was investigated by $\mathrm{Hu}$ et al. (2001), with the objective of demonstrating the occurrence of protein aggregation after induction of focal cerebral ischemia.

Ischemia changes in brain tissue, histologically visible, are observed about $10 \mathrm{~min}$ after ischemia, followed by reperfusion of 24 to $48 \mathrm{~h}$. These changes range from mild neuronal vacuolation, cytoplasmic edema, foam nuclei and presence of irregular chromatin until the presence of late infarction in the ischemic focus region, after longer periods of ischemia and reperfusion (Lipton, 1999; Block et al., 2005; Go et al., 2014). These findings corroborate with those found in this study where histological lesions can be observed in the same regions studied, but with greater severity, mainly in the I + A group, since there was a longer period of reperfusion $(48 \mathrm{~h})$.

In our morphometric evaluation, greater neuronal loss was observed in groups I and $\mathrm{I}+\mathrm{A}$, when compared to the other experimental groups, being slightly larger in the medial region when compared to the lateral region, the latter being less susceptible to ischemia and alcoholism. These changes were also described by Hu et al. (2001) and by Arunadevi et al. (2010), highlighting the presence of mitochondrial edema and increased endoplasmic reticulum volume, foci of edema in the neuropil, as well as the presence of sary lysosomes, digestive vacuoles and condensation of the chromatin of the neural cells, suggesting irreversible lesion of the neural cells.

Cerebral edema is a potentially fatal complication of cerebral infarction and was histologically observed in the three areas under study, with the most prominent layers of lateral and dorsolateral cortex and medial part of the striatum only in groups I and I + A. Zoppo and Mabuchi (2003) and Block et al. (2005) reported functional alterations in brain capillaries after ischemia. This allows the release of vascular contents in the ischemic region, with the presence of leukocyte infiltration in the neural tissue, favoring the pathogenesis of ischemia.

Wu et al. (2018), demonstrated that the death of cortical neurons and the striatum in the penumbra zone, after focal ischemia for two hs, followed by reperfusion of $24 \mathrm{~h}$; 
involves apoptosis characterized by DNA damage and activation of CASPASE 3. Therefore, the choice of CASPASE 3 as a sign of the apoptotic neural lesion mechanism for our study was due to the fact that it is an effector caspase, thus, a common pathway of cell death by (2) and (2), respectively, of the two types of cerebral ischemia, both of which are intrinsic and extrinsic.

The CASPASE 3-positive cell count among the groups we evaluated after analysis in the cortex and striatum showed that this protein was expressed in all regions analyzed, but its expression was higher in the medial region of the dorsolateral cortex and in the lateral regions and super-medial striatum in agreement with the results described by Tirapelli et al. (2010). When we evaluated groups A and I + A, we observed that the expression of CASPASE 3 in group A was slightly higher when compared to groups $\mathrm{C}$ and $\mathrm{S}$, suggesting a small degree of cell death due to apoptosis in these animals, caused by the direct action of alcohol.

We also observed that a greater expression of CASPASE 3 was observed for the animals of group I + A when compared to group I, demonstrating that when associated with ischemia, the injuries caused by alcoholism are even more severe.

The BCL2 family of proteins plays a crucial role in the regulation of intracellular signals of apoptotic transduction. It is important to note that this high expression of BCL2 in the three areas, mainly in groups I and I + A, however, was not as high as CASPASE 3. This can be explained because the lower expression regions of CASPASE 3 are sites corresponding to ischemic focus, a site where there is a high index of the mechanism of cell death due to necrosis, in an initial phase after the instituting of the ischemic insult.

Among the findings of recent studies of our group, neuronal loss was also higher in the medial striatum region of the animals of groups I and I + A and the protein expression of CASPASE -3 was higher than that of XIAP in groups I and I + A for both proteins. (Carvalho et al., 2016; Schiavoni et al., 2018).

The studies of Dharap et al. (2009) indicate that focal ischemia significantly alter the temporal expression of many miRNAs, which could be controlling the transcription and translation of mRNA and thus the resulting pathophysiology of stroke. Future research will show whether modulation of specific miRNAs may be a therapeutic option to prevent postischemic pathophysiological events and/or to promote plasticity and regeneration.

Our study also used serum miRNA-21 assessment after 90-min ischemia and 48-h reperfusion. Studies by Chan et al. (2005) have shown that the absence of apoptosis is directly related to miRNA-21. The aberrant expression of this miRNA blocks the expression of gene products that promote the normal differentiation of glial cells, or induce apoptosis, keeping the cells in a continuous proliferative state. Thus, miRNA-21 was classified as an antiapoptotic factor. Several reports have demonstrated the role of specific miRNAs in neuronal differentiation, neurogenesis, neural cell specification, and in action during neurodevelopment.

Several studies have examined the correlation between miRNAs and cerebral ischemia, increasing the knowledge and importance of the subject (Dharap et al., 2009; Buller et al., 2010; Liu et al., 2010). Among the miRNAs studied, the miRNA-21 acts as a trivial marker for various diseases and plays a critical role in different types of cerebral vascular accidents: hemorrhagic or ischemic, for example. Inhibition of miRNA-21 promotes the reduction of cell proliferation, induction of apoptosis and inhibition of disease progression. Thus, miRNA-21 may serve as a potential marker for prognosis, diagnosis and 
a therapeutic target. Studies have reported high levels of miRNA-21 in stroke but more studies are needed to better understand their behavior (Panagal et al., 2019; Silva et al., 2019).

As an anti-apoptotic factor in several biological systems, miRNA-21 was studied by Buller et al. (2010) after cerebral ischemia in rats. In situ hybridization revealed high expression of miRNA-21 in the neurons of the border ischemic zone, and analysis of gene expression by real-time PCR showed that after ischemia, there was an approximately threefold increase in miRNA-21 levels in neurons isolated from the border ischemic zone, observed after laser microdissection, compared to contralateral homologous neurons, two and seven days after reperfusion. Therefore, the results indicated that high miRNA-21 expression protects against ischemic neuronal death and that the low expression of the FASinducing ligand (FASL), a member of the tumor necrosis factor- $\alpha$ family, the miRNA- 21 , probably mediated its neuroprotective effect.

Compared to previous findings, our results showed low gene expression of miRNA21 in the blood of ischemic animals; however, was higher in the animals of groups A, I and $\mathrm{I}+\mathrm{A}$. The increased expression of miRNA-21 in the animals of groups I and I + A may indicate its protective function to the cells of ischemic death. We know, however, that ethanol suppresses the action of miRNA-21. However, its expression is very close to that observed for the animals of group I, as well as its expression is also higher in the animals of group A compared to groups $\mathrm{C}$ and $\mathrm{S}$. These results suggest that the low suppressive action of ethanol does not $(20 \%)$, which was not enough to induce its suppressive function on the miRNA-21.

In another study, miRNA-21 was significantly upregulated in mice fed ethanol $24 \mathrm{~h}$ after partial hepatectomy despite the inhibition of cell proliferation, suggesting a more complex role for this miRNA (Juskeviciute et al., 2016). The importance of understanding miRNA expression changes related to alcohol abuse and dependence can be appreciated by the impact of various studies on various diseases. Early diagnosis and treatment of complex diseases such as alcoholism may benefit from miRNAs studies, designated to identify disease-specific molecular markers, which provide a fingerprint of the condition; or to identify potential therapeutic targets.

A better understanding of ethanol-induced miRNA expression changes may also provide accurate means to diagnose various consequences of alcohol abuse as well as to identify possible therapeutic strategies for treating the condition and/or consequences of ethanol abuse (Miranda et al., 2010).

In this study, alcohol alone did not cause morphological lesions and increase in significant protein and gene expression; however, when associated with ischemia, lesions were usually more severe than those of group I animals (Figures 4 and 5).

Therefore, histopathological changes were observed in the three analyzed areas of brain tissue, with a significant loss of neuron number. The expression of CASPASE 3 was higher when compared to BCL2 expression in the animals of groups I and I + A. The protein expression of CASPASE 3 was greater in the penumbra area, where apoptosis is more intense, and in regions where the histopathological changes were more intense, the expression of BCL2 was lower. Low serum gene expression of miRNA-21 was observed in all experimental groups; however, was slightly higher for the animals of groups A, I + A and especially I, therefore, their expression was not suppressed by the action of ethanol. 
The I and I + A groups were the groups that presented more histopathological changes, with a greater neuronal loss and cerebral edema, and a greater expression of CASPASE 3, suggesting that ischemia and alcoholism, when associated, can cause considerable injury and damage to the brain tissue. Further studies are needed to better understand the mechanism of ischemia in the injured brain area and in the penumbra area.

\section{ACKNOWLEDGMENTS}

No specific institutional funding was used for this research.

\section{CONFLICTS OF INTEREST}

The authors declare no conflict of interest.

\section{REFERENCES}

Arunadevi R, Ramteke VD, Kumar S, Shukla MK, et al. (2010). Neuroprotective effect of s-methylisothiourea in transient focal cerebral ischemia in rat. Nitric Oxide. 22: 1-10.

Bala S, Szabo G. (2012). MicroRNA Signature in alcoholic liver disease. Int. J. Hepatol. 498232.

Block F, Dihné M and Loos M (2005). Inflammation in areas of remote changes following focal brain lesion. Prog. Neurobiol. 75(5): 342-365.

Buller B, Liu X, Wang X and Zhang RL (2010). MicroRNA-21 protects neurons from ischemic death, FEBS J. 277: 4299-4307.

Carlotti Jr CG, Colli BO and Kazuo JY (2001). Avaliação da isquemia cerebral pela respiração mitocondrial, Arq. Neuro-Psiquiatr. 59: 365-371.

Carvalho CAM, Tirapelli DPC, Rodrigues AR, Lizarte Neto FS, et al. (2016). Morphological and immunohistochemical analysis of apoptosis in the cerebellum of rats subjected to focal cerebral ischemia with or without alcoholism model. Acta Cir. Bras. 31(9): 629-37.

Chaitanya GV, Schwaninger M, Alexander JS and Babu PP (2010). Granzyme-B is involved in mediating post-ischemic neuronal death during focal cerebral ischemia in rat model. Neuroscience. 165: 1203-1216.

Chan JA, Krichevsky AM and Kosik KS (2005). MicroRNA-21 is an antiapoptotic factor in human glioblastoma cells. Cancer Res. 65(14): 6029-6033.

David CA, Prado R and Dietrich WD (1996). Cerebral protection by intermittent reperfusion during temporary focal ischemia in the rat. J. Neurosurg. 85(5): 923-928.

Dharap A, Bowen K, Place R, Li LC, et al. (2009). Transient focal ischemia induces extensive temporal changes in rat cerebral MicroRNA. J. Cerebr. Blood F. Met. 29: 675-687.

Ferrer I, Friguls B, Dalfó E, Justicia C, et al. (2003). Caspase-dependent and caspase independent signalling of apoptosis in the penumbra following middle cerebral artery occlusion in the adult rat. Neuropathol. Appl. Neurobiol. 29: 472481.

Ginsberg MD (2003). Adventures in the pathophysiology of brain ischemia: penumbra, gene expression, neuroprotection: the 2002 Thomas Willis lecture. Stroke. 34: 214-223.

Go AS, Mozaffarian D, Roger VL, Benjamin EJ, et al. (2014). Heart disease and stroke statistics: update: A report from the American Heart Association. Circulation. 129: e28-292.

Hachimi-Idrissi S, Van Hemelrijck A, Michotte A, Smolders I, et al. (2004). Postischemic mild hypothermia reduces neurotransmitter release and astroglial cell proliferation during reperfusion after asphyxial cardiac arrest in rats. Brain Res. 1019: 217-225.

$\mathrm{Hu}$ B, Janelidze S, Ginsberg MD, Busto R, et al. (2001). Protein aggregation after focal brain ischemia and reperfusion. J. Cerebr. Blood Flow Metab. 21: 865-875.

Juskeviciute E, Dippold RP, Antony AN, Swarup A, et al. (2016). Inhibition of miR-21 rescues liver regeneration after partial hepatectomy in ethanol-fed rats. Am. J. Physiol. Gastrointest. Liver Physiol. 311(5): G794-G806.

Kamestu Y, Osuga S and Hakim AM (2003). Apoptosis occurs in the penumbra zone during short-duration focal ischemia in the rat. J. Cerebr. Blood Flow Metab. 23: 416-422.

Koiosumi J, YoshidaY, Nakazaa T and Oneda G (1986). Experimental studies of ischemic brain edema. 1. A new experimental model of cerebral embolism in rats in wich recirculation can be introduced in the ischemic area. $J$. Stroke. 8: 1-8.

Kosik KS and Krichevsky AM (2005). The elegance of the microRNAs: a neuronal perspective. Neuron. 47: 779-782. 
Lee RHC, Lee MHH, Wu CYC, Couto E Silva A, et al. (2018). Cerebral ischemia and neuroregeneration. Neural Regen. Res. 13(3): 373-385.

Lipton P (1999). Ischemic cell death in brain neurons. Physiol. Rev. 79: 1431-1568.

Liu DZ, Tian Y, Ander BP, Xu H, et al. (2010). Brain and blood microRNA expression profiling of ischemic stroke, intracerebral hemorrhage, and kainate seizures. J. Cerebr. Blood Flow Metab. 30(1): 92-101.

Love S. (2003). Apoptosis and brain ischemia. Prog. Neuro-Psychoph. 27: 267- 28.

Miranda RC, Pietrzykowski AZ, Tang Y and Sathyan P (2010). MicroRNAs: master regulators of ethanol abuse and toxicity? Alcohol Clin. Exp. Res. 34(4): 575-587.

Panagal M, Biruntha M, Vidhyavathi RM, Sivagurunathan P, et al. (2019). Dissecting the role of miR-21 in different types of stroke. Gene. 681: 69-72.

Rami A (2003). Ischemic neuronal death in the rat hippocampus: the calpain- calpastatin- caspase hypothesis. Neurobiol. Dis. 13(2): 75- 88.

Reilly MT, Noronha A and Warren K (2014). Perspectives on the neuroscience of alcohol from the National Institute on Alcohol Abuse and Alcoholism. Hand. Clin. Neurol. 125: 15-29.

Schiavoni VS, Silva JPD, Lizarte Neto FS, Assis MLC, et al. (2018). Morphological and immunohistochemical analysis of proteins CASPASE 3 and XIAP in rats subjected to cerebral ischemia and chronic alcoholism. Acta Cir. Bras. 33(8): 652-663.

Silva J, Lizarte Neto FS, Cirino MLA, Carvalho CAM, et al. (2019). Analysis of Caspase-9 protein and microRNAs miR-21, miR-126 and miR-155 related to the apoptosis mechanism in the cerebellum of rats submitted to focal cerebral ischemia associated with an alcoholism model. Arq. Neuro-Psiquiatr. 77(10): 689-695.

St. Clair HR St (1991). Recognizing alcoholism and its effects: a mini-guide. Karger; Basel.

Tirapelli DPC, Carlotti Junior CG, Leite JP, Tirapelli LF, et al. (2010). Expression of HSP70 in cerebral ischemia and neuroprotective action of hypothermia and ketoprofen. Arq. Neuro-Psiquiatr. 68(4): 592-596.

Wu MY, Yiang GT, Liao WT, Tsai APY, et al. (2018). Current Mechanistic Concepts in Ischemia and Reperfusion Injury. Cell Physiol. Biochem. 46(4): 1650-1667.

Zoppo GJ and Mabuchi T (2003). Cerebral microvessel responses in focal ischemia. J. Cerebr. Blood Flow Metab. 23(8): 879-894. 